\title{
Conception et construction des turbomachines de grandes dimensions Les turbines Francis de la centrale de Tucurui (Brésil) Design and construction of large turbines The Francis turbines of the Tucurui station (Brasil)
}

\author{
A. Sauron \\ Chef du Bureau d'Etudes Turbines \\ Société NeyrPiC, Grenoble \\ G. Caillot \\ Adjoint au Chef du Bureau d'Etudes Turbines \\ Société NeYrPIC, Grenoble
}

\section{Avant-propos}

Les groupes hydroélectriques se caractérisent naturellement par des avantages d'exploitation exceptionnels :

- excellent facteur d'utilisation (grande robustesse, bonne fiabilité) ;

- rapidité de mise en service et de prise de charge ;

- souplesse d'utilisation.

Lorsque les caractéristiques hydrologiques, combinées au relief, offrent une énergie disponible importante, le recours à des turbomachines de grandes dimensions permet d'ajouter aux qualités précédentes l'intérêt d'une puissance disponible élevée. Ainsi équipe-t-on maintenant une chute de $60 \mathrm{~m}$ avec des machines de plus de $350 \mathrm{MW}$ comme à Tucurui alors qu'il y a une vingtaine d'année, des groupes de l'ordre de $100 \mathrm{MW}$, sous cette même chute, auraient bien illustré le niveau technique de l'époque.

Pour permettre de conserver aux turbines géantes actuelles les qualités traditionnelles des turbomachines, les constructeurs ont développé un volume important de recherches dans tous les domaines et en particulier au niveau des conceptions hydraulique et mécanique, ainsi qu'au niveau de la fabrication.

Citons simplement quelques axes de recherches : - la réduction importante des dimensions pour améliorer l'économie globale des projets tout en élevant le niveau des performances hydrauliques;

- la prévision du comportement de la machine industrielle grâce aux études préalables sur modèle réduit en similitude. Le coût de ces essais est un investissement bien faible au regard des possibilités qu'ils offrent pour prévenir et, le cas échéant, résoudre rapidement les éventuels problèmes d'instabilité ou de cavitation sur les prototypes,
- la détermination beaucoup plus précise des contraintes et des déformations statiques et dynamiques des différentes structures de machine, grâce à une meilleure connaissance des efforts hydrodynamiques permanents et pulsatoires appliqués aux structures et l'analyse de celles-ci par la méthode des éléments finis,

- l'évolution des procédés de fabrication, principalement des techniques de soudage, pour permettre de réaliser en toute sécurité, parfois sur le chantier même, des composants formés de tôles de forte épaisseur, et conduisant à des ensembles d'encombrement et de masse importants.

Avec l'exemple de Tucurui, comme support, nous évoquons les problèmes majeurs qui doivent être maitrisés pour aboutir à la réalisation d'une turbomachine de grandes dimensions, performante et sûre.

Les groupes de Tucurui, quelques chiffres

L'aménagement hydroélectrique de Tucurui se situe sur la rivière Tocantins au nord du Brésil, à environ 300 kilomètres de la ville de Belem.

Il est composé d'un barrage en terre sur la rive droite, d'un déversoir central, et d'une centrale sur la rive gauche de la rivière. La centrale est conçue pour abriter 12 groupes dont 8 correspondent à la première phase de construction, faisant l'objet du présent exposé, les 4 autres étant réservés pour un contrat d'extension ultérieur. Chaque groupe comprend principalement une turbine Francis à axe vertical et un alternateur d'une puissance nominale de 350 MVA, facteur de puissance 0,95 , triphasé $60 \mathrm{~Hz}$, vitesse de synchronisme $81,8 \mathrm{tr} / \mathrm{min}$. Chaque groupe absorbe un débit de l'ordre de $600 \mathrm{~m}^{3} / \mathrm{s}$. 
Les 8 turbines sont de conception Neyrpic, les fabrications étant réalisés pour une part, en France (Neyrpic, Creusot-Loire) et pour une autre part, au Brésil (Mécanica Pesada). Ces turbines sont accouplées à des alternateurs conçus et réalisés par différents constructeurs : Alsthom Atlantique - Jeumont Schneider ( 4 machines), I.E. Brown Broveri ( 2 machines), General Electric do Brasil ( 2 machines).

Les turbines sont alimentées par une conduite forcée de $10,4 \mathrm{~m}$ de diamètre, réduit à $9,85 \mathrm{~m}$ après le coude inférieur et continuant avec ce même diamètre jusqu'au raccordement avec la bâche spirale. Les chutes nettes opérationnelles de la machine varient entre $45 \mathrm{~m}$ et $67,6 \mathrm{~m}$. Sous la chute maximale de $67,6 \mathrm{~m}$ la puissance de la turbine est de $369 \mathrm{MW}$. Trois groupes sont conçus pour fonctionner en compensateur synchrone.

Quelques dimensions:

Bâche spirale :

diamètre d'entrée ......... $\varnothing \quad 9,850 \mathrm{~m}$

encombrement $\ldots \ldots \ldots \ldots \ldots . \ldots \ldots$

Hauteur du distributeur . . . . . $\quad 2,475 \mathrm{~m}$

Roue Francis

hauteur $\ldots \ldots \ldots \ldots \ldots \ldots \ldots . \quad 4,740 \mathrm{~m}$

diamètre $\operatorname{maxi} \ldots \ldots \ldots \ldots \varnothing \quad 8,350 \mathrm{~m}$

masse ............... 230 tonnes

Arbre unique accouplant la turbine à l'alternateur

Entr'axes entre les paliers turbine et alternateur

(1) $1,800 \mathrm{~m}$

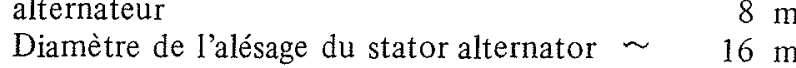

Charge verticale reprise par la pivoterie $2400 \mathrm{t}$

\section{Conception hydraulique}

Les conceptions hydraulique et mécanique d'une machine sont indissociables car il convient de trouver des solutions permettant d'obtenir. simultanément d'excellentes conditions de performance, de réalisation et d'exploitation.

L'aspect hydraulique de cette recherche de la rentabilité maximale s'appuie sur un approfondissement de nos connaissances sur les écoulements dans les turbines et sur les phénomènes de cavitation. Cet effort se traduit en particulier par :

- la réduction des dimensions, l'augmentation de la puissance spécifique et l'adoption de vitesses de rotation élevées tout en maintenant de hautes performances hydrauliques ;

- une meilleure connaissance des efforts hydrodynamiques, permanents et pulsatoires, appliqués aux structures fixes et aux parties tournantes, connaissance acquise par des essais et des mesures, soit sur modèle réduit, soit une machine industrielle.

L'économie d'ensemble des installations de très grandes puissances, équipées de machines géantes, oblige les constructeurs à rechercher une réduction des dimensions. Ces dimensionnements économiques peuvent s'obtenir par réduction du cadre hydraulique (bâche, distributeur), augmentation de la puissance spécifique et adoption de vitesse de rotation élevée.
Les turbines géantes sont donc toujours des machines de hautes performances hydrauliques tant du point de vue des rendements que de la cavitation.

Le Tableau I compare trois aménagements étudiés sous des chutes voisines de 60 mètres :

\begin{tabular}{|l|c|c|c|}
\multicolumn{1}{c}{} & Tableau I \\
\cline { 2 - 4 } \multicolumn{1}{c|}{} & $\begin{array}{c}\text { Mequinenza } \\
\text { (Espagne) }\end{array}$ & $\begin{array}{c}\text { Inga 2 bis } \\
\text { (Zaire) }\end{array}$ & $\begin{array}{c}\text { Tucurui } \\
\text { (Brésil) }\end{array}$ \\
\hline Date & 1958 & 1974 & 1978 \\
\hline$\emptyset$ Roue (mm) & 4300 & 6050 & 8150 \\
\hline Puissance maxi (MW) & 80 & 175 & 360 \\
Chute (m) & 62 & 60 & 66 \\
Vitesse (tr/min) & 150 & 107 & 81,8 \\
ns approximatif & 300 & 320 & 330 \\
\hline
\end{tabular}

Augmentation de la vitesse spécifique et amélioration de la tenue à la covitation

Les puissances spécifiques ont peu varié, par contre, l'effort a porté surtout sur l'accroissement des vitesses de rotation : ns 300 est devenu ns 330 utilisé sous une chute de fonctionnement légèrement plus forte. Par contre, pour conférer toute sécurité vis-à-vis des risques d'usure par cavitation, le sigma limite, définit d'après les observations du modèle, a été volontairement très réduit et la valeur ancienne, voisine de $\sigma=0,33$ pour Mequinenza a été ramenée à $\sigma=0,23$ pour Tucurui.

\section{Réduction des dimensions du cadre hydraulique}

Cette réduction se traduit par des gains économiques concernant :

- l'encombrement extérieur de la bâche et par suite une diminution de la Iongueur de la centrale, avec les gains de Génie Civil qui en découlent,

- certaines structures propres de la turbine, bâche spirale, avant-distributeur, flasques, avec une répercussion directe sur leur poids.

Les progrès accomplis s'illustrent parfaitement à l'aide du dessin comparatif des tracés respectifs des trois machines étudiées (fig. 1,2). On remarque en outre, que cet effort s'accompagne naturellement :

- d'une réduction de la longueur relative du guidage de la grille avant-distributrice (rapport de la longueur des avant-directrices à leur espacement ;

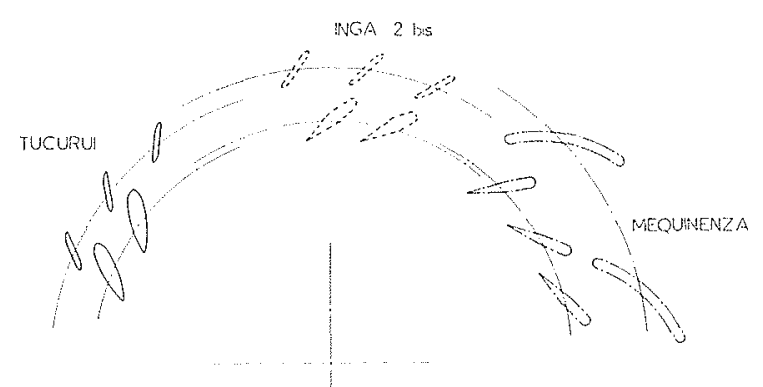

Figure 1. - Tracé hydraulique comparatif de la double grille avant-distributeur - distributeur. 


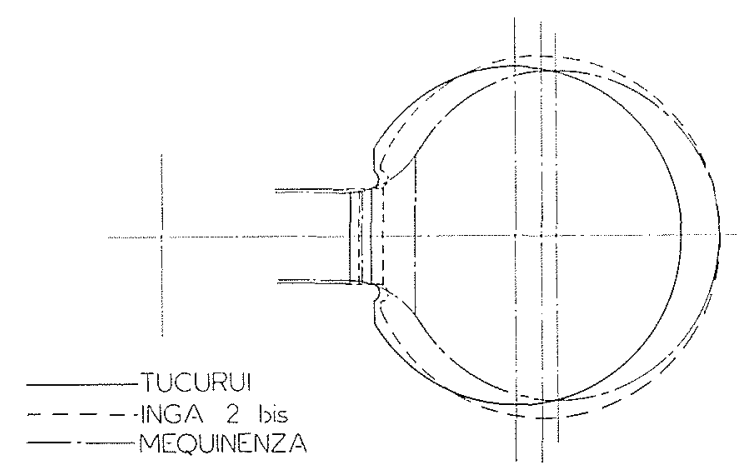

Figure 2. - Tracé hydraulique comparatif des bâches (section méridienne).

- d'angles d'attaque importants;

- d'une imbrication très nette de la double grille constituée par l'avant-distributeur et le distributeur, avec des sections de directrices très supérieures à celles des avant-directrices.

soit un ensemble de problèmes qu'il a fallu résoudre pour pouvoir retenir en toute sécurité ce faible niveau d'encombrement.

\section{Rendements}

On pourrait croire que le rendement des turbines hydrauliques de grandes dimensions a été légèrement sacrifié aux dépens des efforts de rentabilité précédents.

Cela n'est pas le cas, puisque depuis plusieurs années déjà, nous avons apporté la preuve que les rendements des tracés récents atteignent des valeurs très élevées.

C'est cette maitrise des hautes performances qui a permis à Neyrpic de procéder aux exceptionnelles réductions dimensionnelles pour le cadre du Tucurui, tout en améliorant de $1 \%$ le rendement modèle par rapport à Inga 2 bis.

Nous sommes persuadés qu'il existe un optimum économique entre le niveau de performance instantané et la productivité réelle d'une machine. Aussi, est-il bon parfois de sacrifier légèrement le rendement d'une turbine de grandes dimensions pour en faciliter la conception, la fabrication et en accroittre la fiabilité. Dans cet esprit, plutôt que la recherche d'un exploit au niveau des performances, le choix d'un niveau de rendement raisonnable, accompagné de dispositions locales du tracé augmentant la fiabilité de chaque pièce élémentaire de la turbine : roue, avant-distribueur, directrice... semblerait être la voie à suivre. Malheureusement, le contexte de vive concurrence dans lequel se déroulent les réponses aux appels d'offres des aména. gements hydroélectriques ne facilite pas cette démarche.

\section{Sécurité de fonctionnement et connaissance des solli- citations hydrodynamiques}

$\mathrm{Si}$ la conception mécanique des machines doit prendre en compte les amplitudes des efforts hydrauliques pulsatoires, la conception hydraulique doit les réduire au minimum. La diminution de l'amplitude des perturbations hydrodynamiques oriente vers des profils nombreux et effilés tandis que le souci de dimen- sionner les profils pour que leurs fréquences propres se situent au-dessus du domaine des amplitudes maximales des perturbations conduit à des profils épais. D'autres contraintes mécaniques, liées à l'aspect fabrication, sont également à considérer. Les tracés de turbomachines sont donc le fruit d'incessants compromis entre l'hydraulique et la mécanique. Citons quelques exemples de compromis dans la conception des turbines de grandes dimensions :

- l'épaississement des profils de directrices pour permettre le tourillonnement seulement sur deux paliers; - la réduction du nombre des aubes des roues (15 à 17 aubes autrefois pour 11 à 13 maintenant) pour permettre d'utiliser des profils présentant des dimensions plus importantes et offrant une plus grande sécurité vis-à-vis des vibrations;

- le choix des épaisseurs de bord de sortie des aubes qui doivent rester suffisamment minces pour conserver de très bonnes performances, mais en même temps résister aux sollicitations dont ils sont l'objet;

-.. une variation d'épaisseur des aubes de la roue suivant des lois d'évolution à simple courbure pour permettre, si ce mode de fabrication est retenu, une matriçage à partir d'une tôle mère fraisée sur une seule face, etc. La sécurité de fonctionnement des turbines de grandes dimensions suppose une connaissance approfondie des sollicitations hydrodynamiques. Il s'agit en particulier :

- de l'évaluation du comportement dynamique en service des structures fixes et mobiles, afin de prévenir les risques de vibrations dangereuses et de procéder à un dimensionnement correct des structures concernées; - de la connaissance des instabilités d'écoulement et de leurs manifestations, non seulement dans la turbomachine et à l'aval immédiat de la roue, mais également dans l'ensemble de l'aménagement hydroélectrique, incluant conduites forcées et rameaux d'évacuation, ce dernier point restant généralement à la charge du Maitre d'Oeuvre ;

- des prévisions des phénomènes de cavitation, en tenant compte des variations de chute consécutives au grand marnage des retenues. Citons dans le cas de Tucurui, le soin tout particulier apporté à la maitrise, pour les chutes maximales, de la cavitation à l'entrée des aubes, le long du profil et au niveau du raccordement avec la ceinture.

La connaissance de la répartition des pressions le long des aubages constitue un facteur important de progrès dans la conception des turbomachines.

Neyrpic a mis au point un programme de calcul en fluide parfait, supposant l'existence de turbines élémentaires, hypothèse licite dans les zones de fonctionnement voisines des rendements optimaux.

Ce calcul donne la répartition des vitesses dans une grille d'aubes tracées sur une surface de révolution dont la méridienne correspond à la surface moyenne des turbines élémentaires. Ce calcul prend en compte, bien entendu, la variation d'épaisseur des turbines élémentaires de l'entrée à la sortie. Hors de ces zones de fonctionnement optimal, les écoulements deviennent tridimensionnels et nous en étudions alors les particularités à l'aide d'observations sur modèle réduit ; c'est ainsi, par exemple, que nous définissons les répartitions 
de pressions sur chacune des parois des aubes à l'emballement afin de préciser les efforts hydrodynamiques.

Les forces hydrodynamiques pulsatoires pourraient soumettre les organes de la turbine à des cycles de contraintes alternées, de fréquences souvent voisines de certaines des fréquences propres des structures composantes. Seule l'expérimentation sur turbine industrielle et sur modèle réduit permet de connaître l'amplitude et les fréquences de ces sollicitations. Les modèles réduits peuvent alors comporter la mesure des contraintes sur les parties fixes et mobiles, et une analyse assez précise de ces phénomènes fluctuants se fait à l'aide d'enregistrements magnétiques, desquels on peut tirer les densités spectrales d'énergie.

L'étude de la cavitation revêt également un aspect important pour les turbines Francis de grandes dimensions utilisées sous des chutes inhabituelles pour les tracés types retenus. Pour ces machines, les connaissances statistiques des constructeurs, fondées sur la comparaison entre images de cavitation observées sur modèle réduit et usures de cavitation constatées sur certaines turbines, ne peuvent plus s'appliquer sans correction.

Dans ce domaine, nos connaissances de la répartition des pressions, en différents points des aubes, sont à la base de l'estimation des risques d'usures et de la détermination des surfaces qui doivent recevoir une protection d'acier inoxydable.

\section{Conception mécanique}

\section{Disposition générale}

Les groupes sont du type à axe vertical, avec deux paliers-guides (palier alternateur situé sur le croisillon supérieur). Le pivot reprenant les charges verticales excercées sur les parties tournantes s'appuie sur le fond turbine. Le démontage des pièces encombrantes de la turbine, et en particulier de la roue et du flasque, peut s'effectuer à travers l'alésage du stator alternateur.

Les parties fixes de la turbine, situées sous le distributeur et à l'aspiration, sont bétonnées. Toutefois une galerie annulaire, disposée autour du manteau de roue, permet un accès pour le contrôle et l'entretien des tourillons inférieurs des directrices (étanchéité, bagues de guidage). Les directrices reposent sur deux paliers, situés de part et d'autre du conduit hydraulique, elles sont commandées par des servo-moteurs individuels et synchronisées mécaniquement. L'ensemble de la commande du vannage est logé à l'intérieur du flasque supérieur qui se présente comme une structure très largement ouverte (fig. 3).

\section{Les principatux composants}

\section{Bâche spirale et avant-distributeur}

Ce sous-ensemble constitue la structure fixe de plus grandes dimensions. Sa masse est de 420 tonnes environ. La réalisation de structures de cette dimension, effectuée en partie sur le site par suite des limitations de transport, pose des problèmes difficiles de conception et de réalisation.

\section{Principe de réalisation}

Les avant-distributeurs sont réalisés en atelier en autant de parties que le permettent les conditions de transport. Ils sont munis des bavettes de raccordement aux volutes. La soudure des volutes s'effectue en centrale.

La réalisation de la bâche sur le chantier s'accompagne de déformations au cours du soudage, puis du bétonnage. Dans ces conditions, il est nécessaire de reprendre au montage l'usinage des surfaces devant assurer l'appui des flasques et les étanchéités.

L'atelier livre donc des avant-distributeurs non usinés, dont les éléments sont assemblés par soudure au chantier. Après bétonnage de la bâche, les parties fonctionnelles sont usinées.

Avec cette technique de reálisation des avantdistributeurs, les seules opérations d'usinage en atelier consistent à dresser les faces aux coupes et à assurer un repérage précis des différents éléments. Cette manière de procéder évite un tournage coûteux sur une machine de fortes capacités.

Problèmes de conception et de dimensionnement

\section{a) Volute}

Elle est réalisée en tôles d'acier E $36-4$ dont l'épaisseur maximale atteint $34 \mathrm{~mm}$. Ces tôles sont soudées en centrale sur les bavettes intégrées à l'avant-distributeur. Les bavettes sont de même nuance que les viroles, l'épaisseur maximale est de $67 \mathrm{~mm}$.

La réduction des épaisseurs revêt une grande importance économique au niveau des coûts et des délais, car le volume de soudure à déposer est sensiblement proportionnel au carré de l'épaisseur.

Face à ces difficultés, une orientation naturelle semble être le choix d'aciers à très hautes caractéristiques mécaniques (limite élastique de 50 à 70 hbar). Toutefois, la décision n'est pas aussi évidente à prendre car ces aciers posent un certain nombre de problèmes :

- dans le cas des constructions mixtes, ils ne sont d'aucun intérêt lorsque les deux parties à assembler ne sont pas réalisées dans un matériau de ce type. En effet, le niveau des contraintes maximales admissibles est limité par le matériau ayant les caractéristiques mécaniques les plus faibles (cas d'un assemblage avec une partie moulée par exemple);

- le critère des contraintes maximales admissibles n'est pas toujours le critère déterminant, ainsi par exemple, au niveau des avant-directrices, les épaisseurs sont parfois imposées par des impératifs de tenue aux vibrations;

- ces matériaux sont d'une mise en cuvre plus délicate que les aciers courants et entraînent de ce fait des risques de fabrication plus importants. Ils nécessitent, en particulier, une détermination très précise des caractéristiques de soudage (énergie apportée en particulier).

Des détensionnements après formage sont également nécessaires. De nombreux Cahiers des Charges interdisent pour les bâches spirales, l'emploi d'acier dont la limite élastique dépasse 36 hbars.

- Pour permettre d'accroître la résistance, sans augmenter le carbone équivalent, la plupart des aciers 


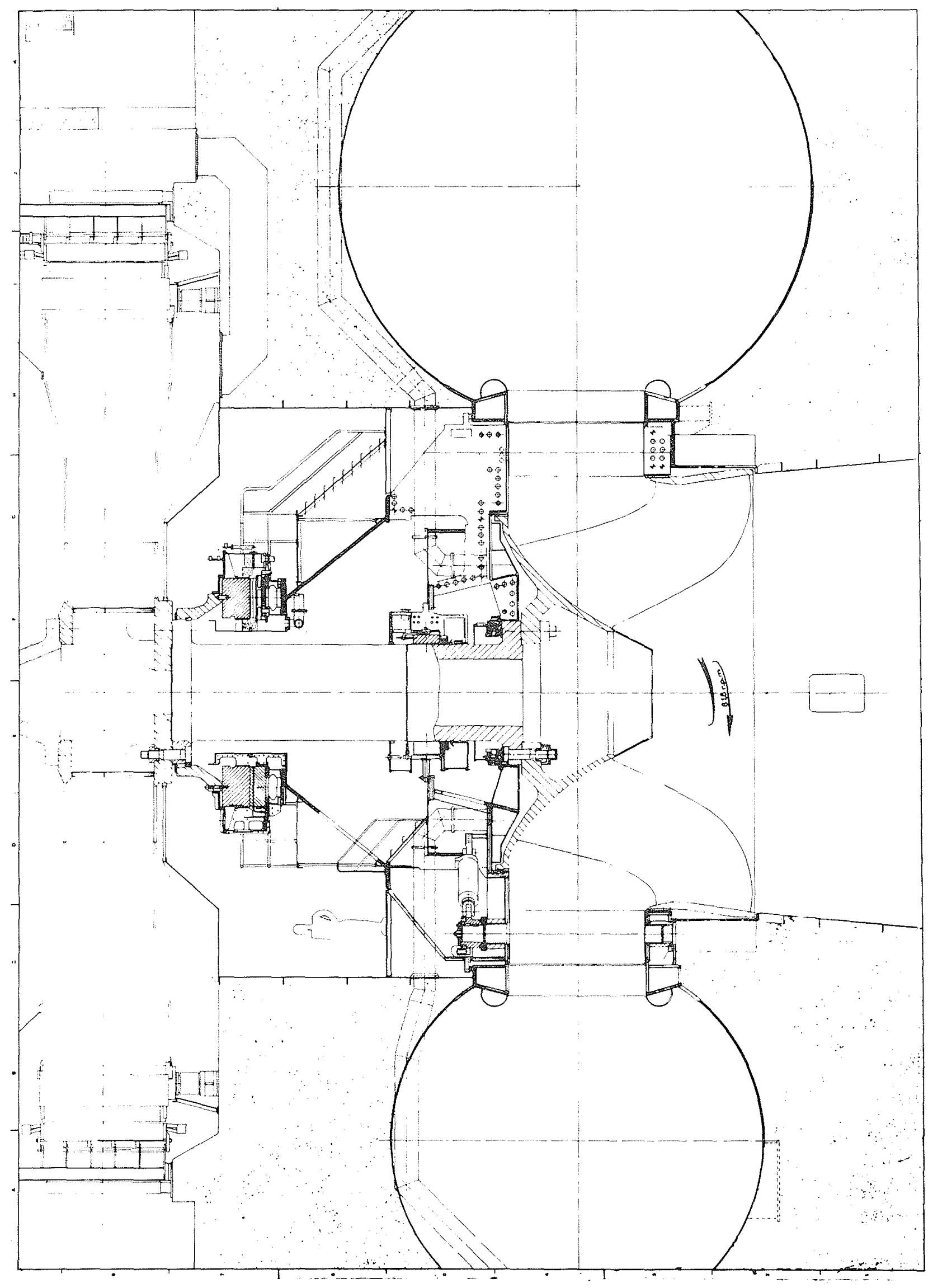

Figure 3. - Coupe d'une turbine de Tucurui - $369 \mathrm{MW}$. 
à haute résistance sont du type trempé et revenu. Il en résulte, en particulier, des pertes de caractéristiques mécaniques :

- si on effectue des traitements thermiques à une température supérieure à $650^{\circ} \mathrm{C}$,

- au niveau de la zone des liaisons par soudure affectée thermiquement.

Ceci diminue d'autant les valeurs à considérer dans les dimensionnements.

- Pour les structures dont le poids intervient essentiellement sur le coût, et non sur d'autres paramètres fonctionnels comme c'est le cas pour les ponts et structures off-shore par exemple, l'intérêt économique d'utiliser des aciers à haute résistance n'est pas évident, l'élévation de prix par rapport aux aciers classiques et les sujétions de mise en cuvre compensant très largement les gains attendus d'une réduction des épaisseurs. Toutefois, cette orientation pourrait être évidemment modifiée par une évolution du marché des tôles mais aussi par les impératifs de construction de machines de grandes dimensions sous chute élevée.

En outre, les procédés de soudage sont à étudier tout particulièrement car ils conditionnent la durée du montage de la bâche.

La détermination précise de l'épaisseur des tôles constituant la volute exige une connaissance approfondie du champ des contraintes s'exerçant dans les différents états possibles et notamment lors de l'èpreuve en pression.

Il $\mathrm{y}$ a lieu en particulier d'étudier avec attention la zone de raccordement avec les anneaux de l'avantdistributeur où des contraintes de flexion, plus ou moins importantes, peuvent prendre naissance selon le degré d'optimisation du point d'attache prévu. Un programme de calcul spécifique, basé sur l'étude du comportement des coques toriques, assure cette fonction d'optimisation.

En outre, dans la zone très particulière du bec de bâche, à la jonction des grandes et petites sections d'enroulement, de fortes contraintes de flexion localisées sont engendrées par les variations rapides de forme. Cependant les capacités d'adaptation du matériau utilisé permettent d'admettre des contraintes locales pouvant dépasser la limite élastique, lors de l'essai en pression.

\section{b) L'avant-distributeur}

Il s'agit d'une structure complexe constituée par deux anneaux-caissons circulaires, reliés entre eux par les avant-directrices formant entretoises. Les avant-directrices sont prolongées à l'intérieur des caissons, afin de les rigidifier et assurer une meilleure diffusion des efforts exercés par la volute de la bâche et les flasques.

Les avant-directrices s'opposent aux déformations des anneaux-caissons, déformations engendrées par l'action de la volute, la pression et les efforts des flasques. Le comportement global des caissons peut s'assimiler à celui d'anneaux toriques, celui des avantdirectrices à des poutres encastrées sur ces anneaux.

L'avant-distributeur comprend 25 avant-directrices, la $26^{\mathrm{e}}$ située dans le bec de bâche étant supprimée. Les profils sont plats avec simplement un taillage des bords d'entrée et de sortie. L'avant-distributeur est construit en atelier en 4 parties qui seront assemblées par soudage sur le chantier. En atelier, on réalise un élément à partir de la soudure de structures de base formées des morceaux de guideau supérieur et inférieur et de deux avant-directrices soudées sur ceux-ci.

Compte tenu de la taille des machines de Tucurui, une étude par éléments finis de l'avant-distributeur a été effectuée afin d'analyser le comportement statique de cette structure complexe. Cette analyse a été conduite par le programme de calcul Neystruc developpé par Neyrpic et traitant du comportement tridimensionnel d'éléments de plaque triangulaire et rectangulaire. Le chargement pris en compte est également tridimensionnel. L'avant-distributeur étant assimilé à une structure cyclée, seul un pas de structure comprenant une avant-directrice et les morceaux d'anneaux correspondants est à considérer. La condensation de la structure étudiée n'introduit aucune approximation car le programme prend automatiquement en compte les conditions aux limites permettant de relier les efforts et les déformations des deux sections latérales limitant le pas.

Cette étude a permis d'analyser le champ des contraintes sous charge et en particulier, de mettre en évidence, l'intérêt de nervures intermédiaires reliant la partie de l'avant-directrice située dans le caisson et le bord extérieur de ce dernier, afin de mieux répartir les efforts provenant de la volute; disposition qui a été retenue sur les machines industrielles.

Aux sollicitations quasi statiques exercées par la volute et les flasques, peuvent se superposer des sollicitations hydrodynamiques pulsatoires au niveau des profils.

$\mathrm{Si}$ on analyse les évolutions dimensionnelles des avant-distributeurs en fonction de la chute, on constate qu'à diamètre d'avant-directrice identique, lorsque la chute diminue, la hauteur du distributeur augmente et l'épaisseur des avant-directrices diminue. Ces dernières évolutions vont toutes les deux dans le sens d'un abaissement des frêquences propres. On conçoit dans ces conditions que les avant-directrices soient de plus en plus exposées à des contraintes vibratoires sous l'action de forces excitatrices pulsatoires liées soit à des décollements sur l'arête d'entrée, soit à des lachers de tourbillons de Karman au niveau de l'arête de sortie.

Ces sollicitations pulsatoires ont pour origine les fluctuations de portance et de couple dues à l'écoulement le long de l'avant-directrice. Ces fluctuations, par suite d'un phénomène d'autoexcitation, s'attachent à une fréquence propre de l'avant-directrice (dans la pratique, le premier mode de flexion). Elles peuvent atteindre une amplitude suffisante pour provoquer des contraintes dynamiques importantes.

Rappelons que pour des cas simples, tels qu'un cylindre placé dans un écoulement perpendiculairement à ses génératrices, il se forme dans le sillage du profil des tourbillons réguliers et un spectre d'excitation se résumant à une fréquence unique. On peut alors relier la fréquence de lacher des tourbillons $\left(f_{K}\right)$ à la vitesse d'écoulement $(V$ ) et à la dimension transversale du profil (e) par une relation du type : 


$$
f_{K}=S \frac{V}{e}
$$

où $S$ représente le paramètre adimensionnel de Strouhal, constant pour des profils d'un même type en similitude géométrique.

Lorsqu'on passe à des formes de profil complexes, telles que celles des avant-directrices, on relève plusieurs difficultés :

- La fréquence des lachers de tourbillons ne dépend plus simplement de la dimension transversale du profil, mais aussi de la forme générale du profil et du bord de fuite en particulier ;

- Le spectre des excitations est généralement plus étalé et c'est ce que nous avons enregistré, lors d'essais récents, sur un modèle d'avant-directrice pour lequel nous avons analysé expérimentalement les fluctuations de vitesse dans le sillage immédiat du bord de fuite. Nous avons observé que tous les spectres présentaient globalement un palier jusqu'à un nombre de Strouhal de 0,3 à 0,35 , puis une décroissance relativement linéaire en échelle logarithmique. Dans le cas d'un spectre étalé, on peut ainsi définir un nombre de Strouhal de référence en caractérisant la fréquence la plus élevée des sollicitations d'amplitude notable.

L'ampleur de la gravité du phénomène d'autoexcitation dépend :

- de la forme du profil de sortie des avant-directrices qui conditionne en partie la forme du spectre des excitations et leurs amplitudes ;

- de la position de la fréquence propre de flexion, par rapport au spectre des excitations.

Signalons en outre, l'apparition de fissurations sur des groupes en exploitation, fissurations qui peuvent être liées à des défauts initiaux inhérents à un manque de rigueur dans les opérations de fabrication et de montage des avant-distributeurs. Outre la parfaite exécution des contrôles habituellement prévus, il est nécessaire de limiter au minimum les contraintes résiduelles, car elles se superposent aux contraintes statiques sous charge et aux contraintes dynamiques. Dans cet esprit, certaines dispositions sont à prendre, citons :

- la mise au point d'une gamme de fabrication permettant d'effectuer les soudures avec le minimum de bridage et en contrôlant les déformations;

- la conduite des traitements thermiques avec des paramètres strictement contrôlés par des thermocouples placés directement sur l'élément à traiter, en contrôlant les parties les plus minces et les plus épaisses;

- le meulage partiel des congés de raccordement des avant-directrices avec les anneaux de l'avant-distributeur dans les zones sensibles.

La conception des avant-distributeurs doit donc prendre en compte :

- les efforts statiques en service ;

- la réponse dynamique des avant-directrices aux excitations engendrées par l'écoulement ;

- les facteurs de concentration de contrainte liés aux formes ;

- la limite de fatigue du matériau dans l'eau.
En outre, les procédés de fabrication en atelier et sur le site doivent être adaptés pour réduire au minimum les contraintes internes et écarter les risques de micro. fissuration.

\section{Le distributeur}

Il comporte 26 directrices synchronisées mécaniquement entre elles par des biellettes et commandées par des servomoteurs individuels logés dans le flasque supérieur. Les directrices tourillonnent sur deux paliers (un dans chaque flasque) équipés de bandes autolubrifiantes en Téflon chargé à $15 \%$ de fibres de verre. L'accès à ces paliers est possible par l'extérieur, permet. tant ainsi un entretien aisé.

\section{Directrices}

Le profil utilisé, de grande largeur pour permettre de limiter l'appui à deux paliers, est partiellement creux. Pour s'adapter au mieux aux conditions locales d'approvisionnement et de fabrication, deux types différents de réalisation ont été retenus. L'un correspondant aux fabrications brésiliennes, consiste à créer la directrice par soudage d'un axe forgé traversant, portant les tourillons, sur lequel viennent s'attacher d'une part, un nez constitué par deux tôles embouties reliées, côté entrée, par un profil rond et d'autre part, une tôle taillée reconstituant la queue du profil. L'autre réalisation, exécutée en France, consiste à utiliser un élément moulé portant tourillons et bord de sortie du profil puis de lui adjoindre par soudure un nez constitué par un embouti unique.

Cette conception a été rendue possible grâce à une étude approfondie de cette structure par éléments finis. Les cas de charges correspondant à la reprise des efforts de pression ainsi qu'à un éventuel effort de coincement ont été analysés afin de vérifier le comportement du profil tant au niveau des contraintes que des déformations (flèches latérales, déformations locales du profil creux, vrillage sous l'action des couples hydrauliques et du frottement dans le palier inférieur). Cette ana. lyse, par éléments finis, a ainsi permis d'adopter sans risque une conception de ce type. Elle a aussi permis de mettre au point une méthode de dimensionnement plus rapide, qui pourra être utilisée dans les applications futures, permettant ainsi d'éviter un recours systématique aux éléments finis lorsque cela n'est pas nécessaire.

Signalons qu'à l'occasion de cette étude, nous avons analysé, par éléments finis, des solutions avec un tourillon ne traversant pas le profil mais séparé en deux parties, chacune venant s'encastrer, dans le profil creux sur une hauteur de $340 \mathrm{~mm}$. Nous avons très rapidement résolu les problèmes de dimensionnement de cette zone d'encastrement, mais par contre nos calculs ont fait apparaître :

- un accroissement important des déformations d'ensemble par rapport à celles obtenues avec la théorie des poutres, alors que la solution avec tourillon traversant la vérifie bien ;

- des déformations préoccupantes du profil lui-même, sous les actions de pression, lorsqu'on s'éloigne des encastrements. 
Après avoir examiné différentes solutions de renforcement, nous sommes arrivés à la conclusion que ces inconvénients ne pouvaient être estompés que par un nervurage très important. Nous n'avons donc pas retenu ce type de solution.

\section{Flasques}

a) Le flasque inférieur de conception classique s'appuie sur un anneau de scellement soudé sur l'avant-distributeur d'un part, et reposant sur la bride de l'aspirateur d'autre part.

b) Le flasque supérieur relève par contre d'un niveau de conception beaucoup plus élaboré.

Il se caractérise par :

- une structure ouverte permettant de loger la commande du vannage, y compris les servomoteurs. L'accès à cette commande est possible entre les nervures disposées entre chaque directrice. Ces nervures relient la partie supérieure du flasque à la partie inférieure en contact avec le conduit hydraulique ;

- un support de palier, constitué par une virole tronconique soudée sur la partie inférieure du flasque. Ce type de réalisation tout en assurant une excellente rigidité permet de découpler les dilatations au niveau du palier des déplacements axisymétriques radiaux du flasque :

- un cône support de pivot, posé et centré sur le flasque au montage, puis assemblé au flasque par une soudure de résistance, la séparation ultérieure de ces deux éléments n'étant pas utile pour l'entretien du groupe. L'inclination du cône est conçue pour optimiser le chargement du flasque sous l'action des charges verticales.

Pour mettre au point une structure de cette importance, nous avons très largement utilisé la méthode des éléments finis. L'ensemble de la structure ayant été maillé en éléments de coque chargés tridimensionnement, nous avons ainsi vérifié l'ensemble du dimensionnement, tant au niveau des contraintes que des déformations. Ces calculs ont particulièrement mis en évidence le mode de sollicitation des nervures et montré précisément l'intérêt des goussets de répartition au niveau de l'attache des nervures sur la partie supérieure du flasque.

\section{Commande du vannage}

Chaque directrice est reliée directement à un servomoteur de commande qui en accompagne les mouvements en toute circonstance. Par contre, un organe fusible (doigt de cisaillement) est prévu entre la synchronisation et le levier de commande de la directrice. Cette conception permet de contrôler, grâce au servomoteur, les mouvements de la directrice lorsque celle-ci a été accidentellement désolidarisée du reste du distributeur (fermeture sur un corps étranger...).

Le dimensionnement des servomoteurs et du cercle de synchronisation a été vérifié pour les fonctionnements transitoires. Le cas d'une directrice désolidarisée du reste du distributeur, à la suite de la rupture du doigt de sécurité, et des efforts hydrodynamiques qui en découlent, sur la directrice désynchronisée ainsi que sur les voisines, a aussi été pris en compte.

Les servomoteurs sont muni d'un verrouillage manuel en position ouverte et fermée pour permettre en toute sécurité les opérations d'entretien. Pour suivre parfaitement les mouvements de la directrice en service, ils sont pourvus à leurs deux extrémités d'articulations à rotule.

La conception du distributeur de Tucurui (directrice sur deux paliers, flasque supérieur ouvert avec support de pivot intégré et incliné de façon à diriger les lignes de forces vers l'avant-distributeur, réduisant ainsi la flexibilité axiale...) constitue une innovation dans le domaine des turbines Francis de grandes dimensions. Une telle progression ne pouvait pas s'envisager dans une étroite coopération entre client et constructeur. Cette collaboration novatrice n'est pas toujours le cas à l'exportation où les solutions traditionnelles sont souvent privilégiées et imposées. Nous en remercions donc d'autant plus vivement Electronorte.

\section{La roue}

La conception et la fabrication des roues sont incontestablement un des éléments fondamentaux de la réussite des turbines géantes. Ceci suppose la maitrise des problèmes de dimensionnement de cet organe, c'està-dire la connaissance des charges permanentes ou fluctuantes d'origine hydrodynamique d'une part, et d'autre part, la maitrise des procédés de calculs sta. tiques et dynamiques précis pour ce type de structure, avec bien sûr en parallèle une évaluation correcte des limites de fatigue des matériaux utilisés. Les problèmes de réalisation reposent essentiellement sur la mise au point d'une gamme appropriée, un choix correct des paramètres de soudage et de traitement thermique, ainsi qu'un suivi minutieux des déformations.

\section{Le dimensionnement}

Nous disposons des programmes par éléments finis permettant le calcul des roues à l'aide d'une schématisation tridimensionnelle en éléments de plaques (Neyrouef). Le calcul statique, disponible depuis plusieurs années, permet de déterminer le champ des contraintes dans l'aube, le plafond et la ceinture, à partir des efforts hydrodynamiques évalués à l'aide d'un calcul en grille d'aubes. La roue de Tucurui a bien entendu été vérifiée à l'aide de cette méthode. Trađitionnellement, ces calculs mettent en évidence deux zones de concentrations des contraintes, l'une à l'attache de l'arête de sortie de l'aube sur le plafond, l'autre à la liaison de l'arête d'entrée de l'aube avec la ceinture.

Les calculs par éléments finis permettent alors de rechercher les modifications de structure locales (épaississement local, adaptation des formes de raccordement) ou globales (notamment formes du plafond) permettant de ramener ces concentrations à des valeurs compatibles avec la limite de fatigue des matériaux.

Dans le cas particulier de Tucurui, le tracé hydraulique retenu a permis d'obtenir un niveau de sollicitations peu élevé au regard des possibilités offertes par les matériaux.

Le calcul dynamique, mis au point par Neypric plus récemment (programme Neyroudy) permet de déterminer, à l'aide d'un maillage moins serré que pour le calcul statique, les fréquences propres de la structure et ses modes de vibration. Rappelons à ce propos, que les fréquences propres de la roue doivent rester assez 
éloignées des fréquences perturbatrices, une résonance franche pouvant entrainer des amplitudes de vibrations sensibles étant donné le faible coefficient d'amortissement réduit des matériaux utilisés $\left(10^{-3}, 10^{-4}\right)$. On constate toutefois que les fréquences propres les plus basses correspondent à des déplacements préférentiels de la ceinture, les modes faisant intervenir essentiellement les aubes apparaissant pour des valeurs de fréquences nettement plus élevées.

\section{Réalisation}

La fabrication des roues de grandes dimensions pose un des problèmes les plus difficiles à résoudre dans la construction des turbines Francis de grande puissance. Audelà d'un certain tonnage, que l'on peut fixer aux environs de 100 à 120 tonnes, il devient nécessaire d'abandonner la solution moulée en une partie.

En effet, même si la construction monobloc restait possible, les risques présentés par la coulée de telles pièces ne sont plus admissibles. Sur ces pièces de grandes dimensions, on a constaté ces dernières années des incidents graves, tels que

- rupture du plafond y compris le plateau d'accouplement,

- séparation quasi totale de la roue selon un plan horizontal par suite d'une interruption accidentelle de l'opération de coulée,

- déformations ou déplacements des noyaux.

Par ailleurs, à l'accroissement des dimensions correspond une diminution du nombre de fonderies capables de couler de telles pièces, d'où incompatibilité avec la recherche de solutions économiques et également avec la cadence de livraison requise (en général 3 à 4 mois entre groupes).

De ce fait pour Tucurui, comme pour nos autres roues de grandes dimensions, nous nous sommes orientés vers une solution consistant à assembler par soudure des éléments séparés :

- plafond moulé en acier ASTM A 643 Grade A, avec attente d'aubes pour raccordement dans un plan horizontal ;

- aubes matricées en E 36-3 (12 aubes par roue);

- ceinture lisse moulée en acier ASTM A 643 Grade A.

La construction en éléments séparés présente de nombreux avantages:

- qualité de la construction : respect des épaisseurs et des dimensions, chacun des éléments peut en effet être usiné séparément ;

- qualité du métal qu'il est plus facile d'assurer sur des composants de masse inférieure ;

- qualité de la similitude hydraulique : lors du montage des aubes, il est relativement facile d'assurer l'homologie avec le modèle : angles et ouvertures ;

- simplification de l'équilibrage de la roue qui est construite à partir d'un plafond et d'une ceinture préalablement usinés et d'aubes que l'on dispose après pesée de manière à réduire le balourd au minimum ; - possibilité d'emploi d'aciers de nuances différentes pour les diverses parties de la roue;

- possibilité de construire la roue sur place si les conditions de transport interdisent la réalisation d'une roue monobloc dans les ateliers du constructeur.
Ces divers avantages expliquent la tendance irréversible vers la construction des roues Fancis de grandes et moyennes dimensions, ainsi que certaines roues de turbines-pompes, à partir d'éléments séparés.

Pour Tucurui, 4 roues sont construires en France, 4 au Brésil.

\section{Plafond moulé}

Afin d'éviter les problèmes posés par une soudure directe en $T$ des aubes sur un plafond lisse (manque d'accessibilité se traduisant par des conditions de travail pénibles et un accroissement du volume des soudures $40 \%$ env. - par ouverture des chanfreins) et malgré l'avantage d'une possibilité d'usinage du plafond avant soudure, présentée par cette solution, le choix s'est porté pour Tucurui sur un plafond avec attente d'aubes.

Dans cette conception, les attentes ont la longueur nécessaire pour permettre de réaliser, dans un plan horizontal, la soudure bout à bout aubes-attentes sur plafond. Cette solution permet de réaliser une soudure de volume réduit, dans de bonnes conditions d'accès ; elle impose par contre un accroissement notable des opérations de meulage. En effet, pour pallier les irrégularités sur le pas et l'épaisseur des attentes, irrégularités inévitables en fonderie, il est nécessaire de prévoir des surépaisseurs pour le raccordement avec l'aube proprement dite, surépaisseurs qui devront être meulées après jonction avec la partie inférieure.

Cet élément reste une pièce délicate de fonderie par sa masse (107t) et sa géométrie.

\section{Aubes matricées}

Cette solution a été mise au point en collaboration avec une firme française spécialisée ; elle est couverte par des brevets. L'approvisionnement de l'ensemble des aubes matricées a été réalisé en France, y compris celles nécessaires à la construction des 4 rolies au Brésil.

Les aubes sont obtenues par formage à partir d'ébauche.

Il convient d'abord de déterminer les dimensions de la tôle capable de cette ébauche. Cette détermination se fait à partir d'une aube modèle réalisée avec un élastomère permettant le développement à chaud de l'aube côté extrados. Ce développement effectué sur aube modèle donne le format de tôle capable de l'ébauche ainsi que les épaisseurs selon un quadrillage prédéterminé. Le procédé utilisé est le suivant :

- emboutissage à chaud de la tôle capable ;

- rechargement automatique inox par feuillard des zones de l'aube soumises à la cavitation ;

- formage final.

Notons que les déformations obtenues au cours du rechargement ont peu d'importance puisque le formage final est effectué en dernier lieu. Après formage, les aubes sont contrôlées sur un chassis permettant de vérifier dimensions, angles et épaisseurs.

\section{Ceinture moulée}

Elle est coulée avec un profil lisse, sans attente au niveau des aubes.

\section{Gamme de fabrication}

\section{a) Préparation des éléments}

- plafond moulé : rechargement des attentes (pro- 
cédé automatique sous flux) et usinage de la tulipe du chanfrein ;

- ceinture moulée : rechargement (semi-automatique ou manuel) des zones où vont venir s'attacher les aubes.

\section{b) Mise en place des aubes}

- plafond retourné et posé à plat (plateau d'accouplement côté sol) ;

- mise en place de la ceinture à l'aide d'un outillage - mise en place des aubes et maintien en position à l'aide d'un outillage ;

- ceinture enlevée.

\section{c) Soudure aube-plafond}

- montage de la roue sur un positionneur à axe horizontal ;

- préchauffage ;

- soudage automatique sous flux, à plat à l'aide de 2 potences diamétralement opposées, la première passe fait l'objet d'un gougeage envers suivi d'une passe de fond manuelle afin d'assurer la santé de la racine de la soudure. Le remplissage des chanfreins s'effectue ensuite de façon alternée, avec une modulation permettant de contrôler les déformations;

- exécution des rayons de raccordement en manuel et semi-automatique.

\section{d) Soudure aube-ceinture}

Procédé analogue à celui de la soudure aube-plafond si ce n'est une segmentation de l'exécution des passes pour permettre d'approcher le galbe du profil par des cordes.

Ce procédé donne d'excellents résultats à condition qu'un soin tout particulier soit apporté :

- au choix d'un couple fil-flux approprié ;

- aux conditions de maintien de pré et post- chauffage ;

- au contrôle des déformations en cours de soudage pour adapter les séquences.

Aucun traitement thermique intermédiaire n'est nécessaire.

\section{Ligne d'arbres}

La conception de la ligne d'arbres constitue un point essentiel de l'étude des groupes hydroélectriques de grandes dimensions. Elle déborde largement de la fourniture électromécanique et implique le Génie civil, ayant à ce niveau des répercussions sur la conception de la centrale.

Pour des groupes équipés de turbines Francis géantes dont le $n s$ est de l'ordre de 200 à 350 , l'importance des couples moteur à transmettre conduit à des arbres de très grand diamètre. Ceux-ci s'avèrent donc très rigides en flexion. On constate ainsi que pour ce type de machine, les rigidités d'appui, au niveau des paliers et principalement, pour ceux situés à proximité de l'alternateur, jouent un rôle essentiel sur les premières vitesses critiques de flexion et le comportement dynamique de la ligne d'arbres. Or, ces rigidités dépendent à la fois des constructeurs électromécaniciens, pour ce qui est des paliers et de leurs supports, mais aussi du Génie civil sur lequel viendront s'appuyer les structures porteuses.

Par intermédiaire de ses paliers, le groupe transmet, en outre, en service, des charges au Génie civil. Ces forces dépendent bien entendu des caractéristiques propres de la turbine et de l'alternateur (balourds mécaniques, efforts hydro-dynamiques, attraction magnétique) mais aussi de la rigidité des paliers (par exemple balourd dû à la déformation de la ligne d'arbres). Elles sont donc liées en partie à des paramètres déperidant du Génie civil. La conception de la ligne d'arbres est donc un point important du projet d'ensemble. A ce titre, les hypothèses de base concernant en particulier la participation du Génie civil aux rigidités doivent être clairement définies lors du choix de la solution définitive. On peut ainsi imaginer les difficultés que pourraient susciter pour un client des projets turbine et alternateur non coordonnés, étudiés séparément et décalés dans le temps.

Pour les turbines de grandes dimensions à axe vertical, trois solutions sont envisageables :

- Groupe avec 2 paliers :

- alternateur en porte à faux (solution dite "parapluie") ;

- palier supérieur situé au-dessus du rotor alternateur;

- Groupe avec 3 paliers.

Examinons rapidement les traits essentiels de ces trois solutions.

Groupe avec 2 paliers, alternateur en porte à faux

Cette solution a pour avantage de simplifier les superstructures de la centrale, aucun effort n'étant à reprendre au-dessus du stator. Elle offre la possibilité de gagner un bout d'arbre en tête de machine et de combiner le palier alternateur avec le pivot, ce qui n'est pas toujours un avantage car cela conduit souvent à faire un palier sur un plus grand diamètre. Par contre, elle exige une très grande rigidité d'appui au niveau du palier alternateur du fait de l'amplification entre l'axe du palier et celui du rotor alternateur. Très sensible à la rigidité de ce palier, elle devient très rapidement instable et ne constitue pas, le plus souvent, une solution saine pour les groupes de grandes dimensions. La reprise des efforts dus à la moitié des pôles en courtcircuit n'est pas en général réalisable dans cette conception, pour les grosses machines.

Groupe avec 2 paliers, dont un au-dessus de l'alternateur

Le rotor alternateur se trouvant situé entre les paliers alternateur et turbine, cette solution tolère des flexibilités d'appui nettement supérieures à celles des groupes type "parapluie" et qui se situent dans un domaine raisonnable de réalisation. Cette solution est en outre beaucoup moins sensible que la solution "parapluie" à une dérive de la rigidité du palier supérieur et est de ce fait beaucoup plus sûre. Elle suppose la reprise des efforts exercés par le palier supérieur au niveau des super structures du Génie civil, la rigidité nécessaire étant en général apportee par un plancher situé au niveau approprié

Cette conception a donné d'excellents résultats pour des groupes tels que :

- Churchill Falls $500 \mathrm{MW}$ sous $313 \mathrm{~m}$ à $200 \mathrm{tr} / \mathrm{min}$; - Cabora Bassa $415 \mathrm{MW}$ sous $103,5 \mathrm{~m}$ à $107 \mathrm{tr} / \mathrm{min}$; - Agua Vermelha $230 \mathrm{MW}$ sous $57 \mathrm{~m}$ à $94,7 \mathrm{tr} / \mathrm{min}$; 
C'est la solution qui a été retenue pour les machines de Tucurui.

Les études de ligne d'arbres, conduites conjointement par Neyrpic et les constructeurs des alternateurs, ont fait apparaître une marge supérieure à $80 \%$ entre la première vitesse critique de flexion et la vitesse maximale de rotation correspondante. L'étude des efforts transmis par la ligne d'arbres à ses paliers a conduit à considérer, pour des fonctionnements transitoires, des charges maximales de l'ordre de $230 \mathrm{t}$ au niveau du palier turbine et de $205 t$ sur le palier alternateur.

\section{Groupe avec 3 paliers}

C'est la solution qui permet de prolonger le domaine de faisabilité des groupes hydroélectriques classiques et donc celle vers laquelle on doit naturellement s'orienter lorsque les deux solutions précédentes ne présentent pas de garanties suffisantes. On pourrait parfois regretter qu'elle soit retenue plus par solution de facilité que par nécessité. Elle s'impose toutefois pour certaines machines très poussées.

Le comportement en torsion des turbomachines du type Tucurui ne présente pas de problème particulier. En effet, compte tenu des rigidités mises en jeu, la première vitesse critique se situe bien au delà de la plage de fonctionnement possible.

L'analyse du comportement axial du groupe doit faire l'objet d'études sérieuses au niveau de la conception. En effet, la roue de la turbine intègre toutes les excitations de l'écoulement, situées principalement dans le domaine des basses fréquences et transmet ainsi des poussées fluctuantes à la ligne d'arbres. Celles-ci sont reprises par l'intermédiaire de la pivoterie et transmise aux parties fixes et au Génie civil. Au niveau alternateur, les sources d'excitation axiales sont faibles : pulsations de pression de la ventilation et certains couplages de mouvement possibles par des variations de frettage de la jante. La turbine constitue indiscutablement une source d'excitation axiale privilégiée et dans ces conditions on peut mesurer l'intérêt, du point de vue partage des responsabilités, de rattacher la pivoterie au lot turbine.

L'étude du comportement axial du groupe fait intervenir de nombreux ensembles :

- pour les parties tournantes : roue, arbre, rotor alternateur (et en particulier sa rigidité interne entre jante et moyeu), appui du grain mobile et grain mobile luimêtne;

- pour les parties fixes : membranes portant les patins, cône support de pivot, flasque supérieur, avant-distributeur et appui sur le béton;

- entre les deux, film d'huile.

La prise en compte de l'ensemble de ces éléments conduit pour des groupes comme ceux de Tucurui à une fréquence propre axiale de l'ordre de $10 \mathrm{~Hz}$. L'ordre de grandeur obtenu montre qu'il s'agit d'une étude dont l'importance ne doit pas êtres sous-estimée.

On peut relever ici, l'un des avantages de l'appui du pivot sur le fond turbine, par rapport au pivot sur croisillon. Le fond turbine présentant une rigidité naturelle importante, l'appui du pivot n'entraîne pas en général de surdimensionnement, par contre pour retrouver cette rigidité sur un croisillon, il faut reconstruire l'équivalent de la rigidité du flasque.

Pour terminer avec la ligne d'arbres, nous signalerons simplement que le palier turbine de Tucurui comporte 8 patins immergés et une réfrigération extérieure. L'arbre creux unique reliant la turbine à l'alternateur est donc lisse et réalisé par soudure des deux plateaux sur le fût central, ces trois éléments étant forgés.

\section{Le pivot}

Le pivot constitue un des points sensibles d'une turbomachine. Il s'agit en effet de supporter une charge de plusieurs milliers de tonnes par un film d'huile de l'ordre de 5 à $7 / 100 \mathrm{~mm}$ d'épaisseur, s'interposant entre des patins et une glace de plusieurs mètres de diamètre. Dans le cas particulier de Tucurui, la charge à reprendre est de $2400 \mathrm{t}$, à l'aise de 16 patins s'appuyant sur un grain mobile de $\phi 4200 \mathrm{~mm}$.

\section{Système d'appui du pivot}

Pour Tucurui, côté patins, la solution avec pivot venant s'appuyer sur le fond turbine a été retenue. A l'occasion de l'analyse des problèmes de ligne d'arbres, nous avons évoqué quelques avantages de cette disposition par rapport à la disposition sur croisillon inférieur de l'alternateur. Nous mentionnerons en outre :

- un rééquilibrage partiel en charge des efforts appliqués par le flasque supérieur sur l'avant-distributeur compte tenu du sens opposé des efforts entre la pression dans le conduit et la charge sur le pivot. Le comportement du flasque peut ainsi être optimisé en choisissant judicieusement le point d'appui du cône support de pivot - une facilité d'accès autour du pivot sans pour autant créer des difficultés pour atteindre et démonter l'ensemble palier-joint.

Les patins sont montés sur des membranes souples remplies d'huile; un circuit les relie entre elles. Les fonctions des membranes sont de compenser :

- les inégalités de flèches en azimut du support de pivot qui ne constitue pas en général une structure axisymétrique, qu'il s'agisse d'un croisillon ou d'un cône avec des ouvertures;

- les déformations d'ensemble du support provoquées par exemple par des mouvements des structures en béton, des fondations, etc.;

- les déplacements lents de la ligne d'arbres qui peuvent se produire à la suite de variations d'efforts sur les patins, variations consécutives à des modifications de la charge de la machine. Les flèches au droit des paliers dépendent en effet des forces appliquées aux parties tournantes. Le basculement de la ligne d'arbres provoque une rotation du plan d'appui des patins.

Cette compensation ne s'accompagne pas de couple de rappel, l'équirépartition des charges sur les patins étant automatique.

Coté grain mobile, afin de s'affranchir d'éventuelles déformations des moyeux des rotors alternateurs, le grain mobile vient prendre appui sur un manchon claveté sur le plateau de l'arbre turbine. 


\section{Les parties actives}

Le pivot de Tucurui supporte une charge de $2400 \mathrm{t}$, bientôt il faudra atteindre 5000 tonnes; aussi Neyrpic $a-t$-il procédé il y a quelques années à un important effort de recherche, dans le cadre d'un contrat DGRST, suivi d'expérimentations sur machine industrielle grâce au concours d'EDF.

Un programme de calcul tridimensionnel, tenant compte des déformations du patin et des fuites latérales, a été développé. Il résoud :

- l'équation de la pression $p(r, \theta)$ dans le film, déduite des équations de Reynolds et de la continuité,

- l'équation de l'énergie dans le film tridimensionnel $r, \theta, z_{h}$,

- l'équation de la chaleur dans le patin en $r, \theta, z_{p}$, - l'équation de la chaleur dans le grain mobile en $r, z_{g}$

- l'équation d'équilibre mécanique du patin, afin d'obtenir les déformations de celui-ci en $r, \theta$,

- l'équation d'équilibre du grain mobile pour obtenir des déformations radiales.

Par ailleurs au cours des itérations du calcul, la position du patin, compte tenu de l'emplacement du point d'appui, s'ajuste à une répartition des pressions correspondante. Il est tenu compte globalement de l'influence de l'espace et du volume d'huile situé entre les patins sur la température du grain mobile. Grâce à cet outil, le comportement en service des parties actives du pivot a pu être mieux appréhendé. En outre pour les pivots futurs de très forte charge, des patins d'un type nouveau, permettant de diminuer les déformations thermiques et d'augmenter les pressions spécifiques, ont pu être mis au point et testés industriellement avec la collaboration d'EDF sur un groupe de Salignac.

Ces problèmes théoriques étant bien maitrisés, nous soulignerons pour terminer le soin qui doit être apporté à la réalisation des parties actives :
- tolérance de planéité de l'ordre de quelques centièmes de $\mathrm{mm}$ pour un grain mobile de $\phi 4,2 \mathrm{~m}$;

- voile dû au défaut de perpendicularité de la face active par rapport à l'axe de la ligne d'arbres à limiter également à quelques centièmes de $\mathrm{mm}$;

ceci afin d'éviter les mouvements dynamiques importants des patins.

\section{Conclusion et perspective d'évolution des moyens de conception}

Grâce aux efforts de recherche engagés ces dernières années, la conception et la réalisation de turbomachines du type de celles de Tucurui, s'effectuent avec toute la confiance souhaitable. Des équipements plus importants peuvent s'envisager sans appréhension particulière.

Il reste toutefois que l'ensemble des études nécessaires à la conception d'une turbine de grande puissance mobilise des moyens humains importants et nécessite un temps d'analyse prolongé. Afin de permettre de mieux optimiser les équipements, il convient de multiplier les analyses comparatives.

Avec les moyens d'étude conventionnels, cela conduirait à une augmentation importante du temps consacré à la conception et au dimensionnement de chaque variante envisagée, ce qui serait incompatible avec les délais de réalisation. Cette démarche est possible en informatisant le processus de conception hydraulique et mécanique. Elle est inéluctable à terme.

Depuis plusieurs années, Neyrpic travaille à la mise au point d'un système de conception et de fabrication assistées par ordinateur. Déjà certaines applications sont opérationnelles. Des liaisons de plus en plus directes se tissent avec la fabrication et l'usinage en commande numérique. Ensemble d'efforts allant dans le sens d'une meilleure compétitivité tout en conservant un haut niveau de fiabilité

\section{Discussion}

Prósident : M. CORDELLE

M. le Président. - Merci beaucoup, M. CAILLOT, de votre présentation tout à fait remarquable. On a eu l'impression tout au cours de votre exposé, que l'on était en train de fabriquer la machine de TUCURUI, ce qui est un tour de force.

J'ouvre la discussion sur cette communication.

M. JEANPIERRE désire insister sur le fait que les grosses machines doivent être étudiées en liaison étroite avec le génic civil pour les questions de rigidité et de report de charges : - les efforts de plus en plus importants doivent passer par une mince couronne de béton pincée entre la volute de la bâche et le puits de l'aspirateur.

- pour obtenir une certaine rigidité au niveau du palier alternateur, on est souvent amené à faire un plancher épais. On peut se poser la question de savoir s'il ne vaudrait pas mieux obtenir la rigidité voulue par de l'acier plutôt que par du béton.
M. JEANPIERRE estime que, plus que par le passé, les études des machines de grandes dimensions doivent être le résultat de concertations poussées entre les projeteurs du génie civil et ceux de l'électro-mécanique.

M. CAILLOT. - est tout à fait d'accord avec ce point de vue. A son avis, la concertation entre génic civil et constructeurs électromécaniciens devrait avoir lieu très en amont, au moment de la conception de la centrale, afin de rechercher une optimisation d'ensemble des constructions. En ce qui concerne le problème des rigidités d'appui au niveau des paliers, et compte tenu de l'importance de ce facteur sur la tenue de la ligne d'arbre des machines de grandes dimensions, le choix du nombre et de la disposition des paliers doit aussi tenir compte des conséquences d'une imprécision dans l'évaluation de cette rigidité. En effet, dans la structure d'appui certains ćléments sont diffi- 
ciles à évaluer ; citons par exemple l'interface entre la structure mécanique et le béton, les éléments d'assemblage d'une structure mécanique démontable, etc.

Examinons dans cet esprit les groupes à deux paliers. Au niveau alternateur, deux dispositions sont possibles :

- alternateur en porte à faux au-dessus du palier supérieur (solution dite parapluie) ;

- palier supérieur situé au-dessus de l'alternateur.

La solution parapluie est intéressante du point de vue génie civil car en général, on dispose naturellement d'une structure béton, rigide, au niveau du plan de pose du stator-alternateur.

Lorsque le palier supérieur est situé au-dessus de l'alternateur, la rigidité d'appui du béton s'obtient en général par la mise en place d'une dalle résistante à ce niveau, ce qui impose parfois des travaux de Génie civil supplémentaires.

Par contre l'analyse du comportement dynamique de la ligne d'arbre dans ces deux solutions met en évidence une très grande sensibilité de la solution parapluie à la rigidité d'appui au niveau du palier alternateur. Les conséquences d'une légère sous évaluation de la flexibilité d'appui, peuvent être catastrophiques dans le cas d'une solution parapluie et pratiquement sans importance si le palier est situé au-dessus de l'alternateur.

Ainsi pour des machines du type de celles de TUCURUI, si l'on avait adopté une solution type parapluie, la flexibilité maximale d'appui (palier + croisillon support + béton) aurait dû être de l'ordre de $5 \mu /$ t. En général, le génie civil participe pour environ $2 \mu / t ; 3 \mu / t$ reste donc pour la structure mécanique. Une flexibilité supplémentaire de $2 \mu / t$ qui résulterait d'imprécision d'évaluation (valeur tout à fait réaliste) conduirait à un effondrement de la stabilité alors qu'elle ne mettrait pas fondamentalement en jeu la sécurité de fonctionnement d'une solution avec palier supérieur pour laquelle la flexibilité d'appui du palier alternateur est de $15 \mu / t$.

M. le Président, qui partage parfaitement la préoccupation de M. JEANPIERRE, est d'accord avec M. CAILLOT : les choix relatifs aux dispositions constructives de la machine et ceux relatifs à l'agencement du génie civil ne peuvent être faits indépendamment l'un de l'autre. Cela étant, il faut reconnaître qu'il y a une réelle difficulté à bien connầtre ce que l'on peut effectivement demander au génie civil aux différents niveaux. Une première démarche pourrait consister à s'efforcer de mesurer la rigidité réelle du génie civil dans des installations existantes.

M. $D O D U$ demande si les calculs des modes de vibrations propres de la roue nécessitent l'exécution de vérifications expérimentales, et si de telles vérifications sont possibles?

C'est également une question du Président.

M. CAILLOT. - répond qu'un étalonnage expérimental des résultats obtenus par le calcul est absolument indispensable.

Dans notre cas, les vibrations propres d'une roue industrielle ont été calculées puis mesurées en atelier. La concordance entre fréquences propres calculées et mesurées a été excellente.

M. ROCHE. - indique que lors de la mise en service de la pompe-turbine biétage réglable du TRUEL, dcs cssais systématiques de flexibilité des appuis seront engagés pour en connaître effectivement les impédances. Pour le génie civil, il $\mathrm{y}$ a aussi ce souci de vérification expérimentale des valeurs calculées.
D'autre part, M. ROCHE pose une question relative à l'aspirateur des turbines de grandes dimensions. L'extrapolation faite à partir de machines plus modestes est-elle acceptable? Ne conviendrait-il pas d'engager des études en vue de diminuer la profondeur du cône d'aspiration et la portée de la voute de la trompette?

M. CAILLOT. - est de cet avis : une optimisation est à faire entre les impératifs du tracé hydraulique, la construction mécanique et la tenue du génie civil. Le problème de la portée des voûtes se pose en particulier avec acuité pour les turbinespompes de grandes dimensions avec un enfoncement important.

M. HAMON. - pose deux questions :

- Pour les directrices à profil creux. ne pourrait-on pas prévoir des renforcements internes pour augmenter la rigidité, et pour améliorer l'inertie, remplir le vide de béton?

- Na-t-on pas envisagé le fractionnement des roues de plus de $8 \mathrm{~m}$ de diamètre dont le transport présente souvent des difficultés considérables?

Pour M. CAILLOT remplir de béton les directrices à profil creux apporte une masse sans augmenter notablement la rigidité. De nombreux ancrages seraient à prévoir pour assurer l'interface béton/acier et il demeurerait l'incertitude d'une perte d'efficacité du remplissage liée à la fissuration du béton. Pour des profils creux de directrices, la solution consistant à prévoir un nervurage interne pour limiter les déformations du profil parait plus sûre.

Les problèmes posés par le transport des roues de grandes dimensions conduisent à la fabrication sur place ou tout au moins à la soudure sur place d'éléments de roue. C'est le cas des roues de CABORA BASSA au Mozambique (diamètre de roue $6,560 \mathrm{~m}$ ).

En réponse à une question de $M . B E D U C C I$, M. CAlLLOT précise que les calculs par éléments finnis qui ont été présentés ont été effectués à l'aide de programmes de calcul mis au point par Neyrpic. En ce qui concerne le calcul des roues, on utilise le caractère cyclé de la structure. Scul un pas constitué par une aube et les structures correspondantes du plafond et de la ceinture est maillé, le programme écrit automatiquement les conditions aux limites qui sont identiques sur les frontières du cyclage.

En réponse à une question de $M$. FINOCHIETTI, M. CAILLOT indique qu'il tient compte de la surpression pour le dimensionnement du distributeur.

M. DELASSUS. - demande quel est le niveau de rendement d'une turbine de la puissance de TUCURUI, quelle est la précision du calcul et s'il y a des garanties à tenir.

M. CAILLOT. - Les tracés hydrauliques se font à deux niveaux : d'abord dans un tracé hydraulique de base qui permet d'avoir un niveau de performance; ensuite il y a des recherches sur modèle réduit qui permettent d'améliorer les performances (rendement, cavitation, stabilité). Compte tenu de la puissance délivrée par ces machines, les clients sont très pointilleux pour les performances et imposent des garanties assorties de pénalités. Pour des roues Francis de ns moyen, les rendements mesurés sur modèle atteignent $94 \%$.

Après avoir remercié $M$. CAILLOT, le Président passe la parole à $M$. CADOT pour un sujet différent de la mécanique puisque M. CADOT, Directeur de l'Entreprise RAZEL Frères, va parler du chantier de construction du barrage de Grand' Maison. 